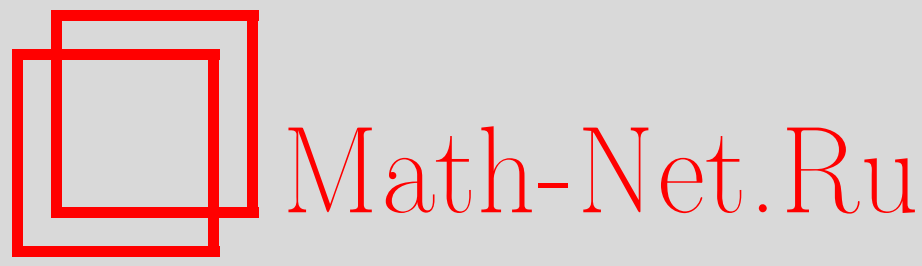

Н. В. Цилевич, Стационарные случайные разбиения натурального ряда, Теория вероятн. и ее примен., 1999, том 44, выпуск 1, 55-73

DOI: https://doi.org/10.4213/tvp597

Использование Общероссийского математического портала MathNet.Ru подразумевает, что вы прочитали и согласны с пользовательским соглашением

http://www . mathnet.ru/rus/agreement

Параметры загрузки:

IP : 3.91 .87 .62

26 апреля 2023 г., 17:18:17

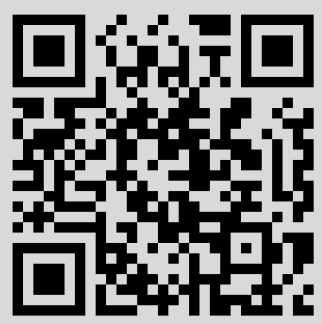




\section{СТАЦИОНАРНЫЕ СЛУЧАЙНЫЕ РАЗБИЕНИЯ НАТУРАЛЬНОГО РЯДА ${ }^{1)}$}

В работе получено описание стационарных случайных разбиений натурального ряда (в эквивалентной постановке - стационарных когерентных последовательностей случайных подстановок) относительно естественного действия бесконечной симметрической группы. Результат дает новую характеризацию распределения Пуассона-Дирихле $P D(1)$ с единичным параметром как единственного инвариантного распределения для некоторого семейства марковских операторов на бесконечномерном симплексе, а также новую характеризацию меры Хаара на проективном пределе конечных симметрических групп.

Ключевые слова и фразы: случайные разбиения, случайные подстановки, стационарное распределение, марковский оператор, распределение Пуассона-Дирихле.

1. Постановка задачи и формулировка результата. Классическая теорема де Финетти описывает все перестановочные последовательности скалярных случайных величин (в терминологии [4] - классы эквивалентных случайных величин), т.е. случайные последовательности, совместные распределения нескольких членов которых не меняются при финитных подстановках элементов. Согласно этой теореме, всякая случайная перестановочная последовательность есть смесь последовательностей независимых одинаково распределенных случайных величин. Имеется большое число различных доказательств этой важной теоремы, среди многих работ отметим [1], [4], [9]. У добно формулировать теорему де Финетти следуюшим образом: всякая эргодическая мера на бесконечном произведении $\prod_{1}^{\infty}[0,1]$, инвариантная относительно действия группы финитных подстановок, есть мера Бернулли.

Та же группа финитных подстановок натурального ряда, т.е. бесконечная симметрическая группа $\mathfrak{S}_{\infty}$, действует естественным образом

${ }^{*}$ С.-Петербургское отделение Математического института им. В. А. Стеклова РАН, ул. Фонтанка, 27, 191011 С.-Петербург, Россия.

1) Работа выполнена при частичной поддержке Российского фонда фундаментальных исследований, грант 96-15-96060. 
на пространстве разбиений натурального ряда $\Pi_{\infty}$. А именно, образ разбиения $\xi \in \Pi_{\infty}$ под действием подстановки $g \in \mathfrak{S}_{\infty}$ есть разбиение $g \xi$ такое, что элементы $i$ и $j$ лежат в одной компоненте разбиения $g \xi$ тогда и только тогда, когда элементы $g^{-1} i$ и $g^{-1} j$ лежат в одной компоненте разбиения $\xi$. Вопрос о перестановочных случайных разбиениях натурального ряда, аналогичный вопросу о перестановочных последовательностях, был поставлен и решен Дж. Кингманом [13] (см. также [12]).

Снабдим пространство $\Pi_{\infty}$ топологией проективного предела пространств разбиений конечных множеств $[n]=\{1, \ldots, n\}$. Пусть $\mathscr{M}\left(\Pi_{\infty}\right)$ - множество всех борелевских распределений на пространстве $\Pi_{\infty}$. Распределение $\eta \in \mathscr{M}\left(\Pi_{\infty}\right)$ называется перестановочным (соответственно, случайное разбиение $\xi \in \Pi_{\infty}$ с распределением $\eta$ называется перестановочным), если $\eta$ инвариантно относительно описанного действия финитных подстановок.

Согласно теореме Кингмана, эргодические перестановочные разбиения натурального ряда параметризуются точками симплекса $\Sigma=\{x=$ $\left.\left(x_{1}, x_{2}, \ldots\right): x_{1} \geqslant x_{2} \geqslant \cdots \geqslant 0, \sum_{i=1}^{\infty} x_{i} \leqslant 1\right\}$ и строятся при помощи следующей конструкции [13]. Пусть $x \in \Sigma$. Рассмотрим распределение $\alpha$ на отрезке $[0,1]$, имеющее счетное число различных атомов с нагрузками $x_{1}, x_{2}, \ldots$ и абсолютно непрерывную составляющую полной меры, равной $1-x_{1}-x_{2}-\cdots$. Пусть $Z_{1}, Z_{2}, \ldots$ - последовательность независимых одинаково распределенных случайных величин с общим распределением $\alpha$. Эта последовательность задает случайное отношение эквивалентности на $\mathbf{N}$ (а следовательно, и случайное разбиение $\xi_{x}$ множества $\mathbf{N}$ на классы эквивалентности), а именно, $i \sim j \Longleftrightarrow Z_{i}=Z_{j}$. Из конструкции следует, что построенное случайное разбиение $\xi_{x}$ перестановочно. Обозначим его распределение через $\bar{P}^{x}$.

Теорема 1 (Кингман [13]). Пусть $\xi$ - перестановочное случайное разбиение натурального ряда. Обозначим через $\xi_{n}$ его ограничение на подмножество $[n] \subset \mathbf{N}$, и пусть $L_{1}\left(\xi_{n}\right) \geqslant L_{2}\left(\xi_{n}\right) \geqslant \cdots-$ размеры компонент разбиения $\xi_{n}$ в невозрастающем порядке. Тогда предель

$$
X_{i}(\xi)=\lim _{n \rightarrow \infty} \frac{L_{i}\left(\xi_{n}\right)}{n}, \quad i=1,2, \ldots,
$$

существуют с вероятностью 1. Для любого $x \in \Sigma$ условное распределение $\xi$ при условии $\left(X_{1}, X_{2}, \ldots\right)=x$ равно $\bar{P}^{x}$. Таким образом, распределение $\bar{\mu}$ перестановочной последовательности $\xi$ задается формулой

$$
\bar{\mu}=\int_{\Sigma} \bar{P}^{x} d \nu(x),
$$

где $\nu$ - распределение вектора $X=\left(X_{1}, X_{2}, \ldots\right)$ на симплексе $\Sigma$.

Перестановочные случайные разбиения используются во многих областях математики и приложений таких, как комбинаторика, теория 
симметрических групп, популяционная генетика. Особо важную роль играет случайное разбиение Ювенса, тесно связанное с мерами Хаара на симметрических группах и распределением Пуассона-Дирихле на симплексе (см. пример 1), которое обладает многими дополнительными симметриями. Целью настоящей работы является новая характеризация этого случайного разбиения.

В связи с задачами теории представлений бесконечной симметрической группы оказывается полезным переформулировать теорему 1 следующим образом. Пусть $\mathfrak{S}_{n}$ - симметрическая группа порядка $n$, т.е. группа всех подстановок множества $[n]$. Канонической проекчией $\pi_{n}: \mathfrak{S}_{n+1} \rightarrow \mathfrak{S}_{n}$ называется отображение, сопоставляющее подстановке $w \in \mathfrak{S}_{n+1}$ подстановку множества $[n]$, получаемую удалением элемента $n+1$ из содержащего его цикла подстановки $w$.

Последовательность $\left(w_{1}, w_{2}, \ldots\right)$ подстановок $w_{i} \in \mathfrak{S}_{i}$ будем называть когерентной, если $\pi_{n} w_{n+1}=w_{n}$ при всех $n=1,2, \ldots$ Обозначим через $\mathfrak{S}^{\infty}$ пространство когерентных последовательностей подстановок, снабженное топологией проективного предела конечных групп $\mathfrak{S}_{n}$ (это пространство введено в работе [11], где оно названо пространством виртуальных подстановок). Пусть $\mathscr{M}\left(\mathfrak{S}^{\infty}\right)$ - множество борелевских вероятностных распределений на $\mathfrak{S}^{\infty}$.

На пространстве $\mathfrak{S}^{\infty}$ определено действие группы $G=\mathfrak{S}_{\infty} \times \mathfrak{S}_{\infty}$. Пусть $\omega=\left(w_{1}, w_{2}, \ldots\right)$ - когерентная последовательность подстановок и $\left(g_{1}, g_{2}\right) \in \mathfrak{S}_{n} \times \mathfrak{S}_{n}$. Положим

$$
\left(\left(g_{1}, g_{2}\right) \omega\right)_{i}= \begin{cases}g_{1}^{-1} w_{i} g_{2}, & \text { если } i \geqslant n \\ \pi_{i}\left(g_{1}^{-1} w_{n} g_{2}\right), & \text { если } i<n .\end{cases}
$$

Обозначим через $K=\left\{(h, h), h \in \mathfrak{S}_{\infty}\right\}$ диагональную подгруппу группы $G$, изоморфную $\mathfrak{S}_{\infty}$. Распределение $\mu \in \mathscr{M}\left(\mathfrak{S}^{\infty}\right)$ называется иентральным, если оно инвариантно относительно действия диагональной подгруппы $K$.

Каждая когерентная последовательность подстановок $\omega \in \mathfrak{S}^{\infty}$ определяет разбиение $\xi(\omega)$ натурального ряда, а именно, $i$ и $j$ принадлежат одной компоненте $\xi(\omega)$, если $i$ и $j$ лежат в одном цикле подстановки $w_{n}$ при достаточно большом $n$. Обозначим через $\Phi: \mathfrak{S}^{\infty} \rightarrow \Pi_{\infty}$ отображение $\omega \mapsto \xi(\omega)$. Действие группы $K$, изоморфной $\mathfrak{S}_{\infty}$, на пространстве $\mathfrak{S}^{\infty}$ согласовано с действием группы $\mathfrak{S}_{\infty}$ на пространстве $\Pi_{\infty}$, т.е. $g \Phi(\omega)=\Phi\left(g^{-1} \omega g\right)$. Поэтому отображение $\Phi$ переводит центральные распределения на $\mathfrak{S}^{\infty}$ в перестановочные распределения на $\Pi_{\infty}$. Причем это соответствие распределений является взаимно однозначным, поскольку центральная мера на симметрической группе $\mathfrak{S}_{n}$ полностью определяется распределением циклового разбиения.

Пусть $P^{x}$ - центральное распределение на пространстве $\mathfrak{S}^{\infty}$, соответствующее эргодическому распределению $\bar{P}^{x}$ на пространстве $\Pi_{\infty}$. В 
терминах случайных подстановок теорема Кингмана может быть сформулирована следующим образом.

Теорема $1^{\prime}$. Пусть $\omega=\left(w_{1}, w_{2}, \ldots\right)$ - чентральная последовательность случайных подстановок. Обозначим через $l_{1}\left(w_{n}\right) \geqslant l_{2}\left(w_{n}\right) \geqslant$ ... длины чиклов подстановки $w_{n} \in \mathfrak{S}_{n}$ в невозрастаюшем порядке. Тогда предельь

$$
X_{i}(\omega)=\lim _{n \rightarrow \infty} \frac{l_{i}\left(w_{n}\right)}{n}, \quad i=1,2, \ldots
$$

существуют с вероятностью 1. Для любого $x \in \Sigma$ условное распределение $\omega$ при условии $\left(X_{1}, X_{2}, \ldots\right)=x$ равно $P^{x}$. Таким образом, распределение $\mu$ чентральной последовательности $\omega$ задается формулой

$$
\mu=\int_{\Sigma} P^{x} d \nu(x)
$$

где $\nu$ - распределение вектора $X=\left(X_{1}, X_{2}, \ldots\right)$ на симплексе $\Sigma$.

Предел $X_{i}(\omega)$ называется относительной длиной $i$-го иикла последовательности $\omega$. Центральное распределение $\mu$ называется насышенным, если оно сосредоточено на последовательностях, у которых сумма относительных длин циклов равна единице. Обозначим через $\phi: \mathfrak{S}^{\infty} \rightarrow \Sigma$ отображение $\omega \mapsto\left(X_{1}(\omega), X_{2}(\omega), \ldots\right)$.

П р и м е 1 . Пусть $w_{1}=e \in \mathfrak{S}_{1}$ - тождественная подстановка. Если уже построена подстановка $w_{n}$, то положим $w_{n+1}=w_{n}(n+1, k)$ с вероятностью $1 /(n+1)$ при $k=1, \ldots, n+1$ (здесь $(i, j)$ обозначает транспозицию, переставляюшую элементы $i$ и $j$ ). Очевидно, что полученная случайная последовательность $\omega=\left(w_{1}, w_{2}, \ldots\right)$ когерентна, центральна, и подстановка $w_{n}$ равномерно распределена на симметрической группе $\mathfrak{S}_{n}$. Распределение построенной последовательности $\omega \in \mathfrak{S}^{\infty}$ называется мерой Хаара на пространстве виртуальных подстановок. Соответствующее случайное перестановочное разбиение натурального ряда называется случайным разбиением Ювенса. Распределение относительных длин циклов для последовательности, распределенной по мере Хаара, есть известное распределение Пуассона-Дирихле $P D(1)$ ([14], см. также независимое доказательство в [8]), т.е. мера Хаара задается формулой (3), в которой нужно положить $\nu=P D(1)$. В частности, мера Хаара не эргодична. Распределение $P D(1)$ сосредоточено на последовательностях с единичной суммой, так что мера Хаара является насыщенной.

Мера Хаара на $\mathfrak{S}^{\infty}$ есть единственное распределение, инвариантное относительно правого (соответственно, левого) действия группы $\mathfrak{S}_{\infty}$ (поскольку при любом $n \in \mathbf{N}$ конечномерная проекция инвариантной меры на $\mathfrak{S}_{n}$ инвариантна относительно всех сдвигов, и, следовательно, является мерой Хаара на $\mathfrak{S}_{n}$ ). Оказывается, условие $\mathfrak{S}_{\infty}$-инвариантности 
является слишком сильным, и мера Хаара на $\mathfrak{S}^{\infty}$ есть единственное распределение, удовлетворяющее более слабому условию стационарности (в классе насыщенных распределений). Опишем эту более общую постановку вопроса, предложенную А. М. Вершиком. Заметим, что образ $R_{g} \mu$ центрального распределения $\mu$ на $\mathfrak{S}^{\infty}$ под действием правого сдвига $R_{g}$ на подстановку $g \in \mathfrak{S}_{\infty}$ уже не является центральной мерой. Однако можно определить ее проекцию $P R_{g} \mu$ на пространство центральных распределений (см. п. 3). Естественно поставить следующую задачу: описать все чентральные распределения $\mu$ такие, что для любой нитной подстановки $g \in \mathfrak{S}_{\infty}$ распределение $\mu$ сохраняется при отображении, являющемся композичией сдвига $R_{g} u$ проекции $P$, т.е. такие, что $P R_{g} \mu=\mu$ для всех $g \in \mathfrak{S}_{\infty}$. Такие распределения будем называть стачионарными. Иными словами, речь идет о центральных распределениях, инвариантных относительно некоторого семейства марковских операторов.

Основным результатом данной работы является описание всех стационарных центральных распределений.

Теорема 3. Эргодические чентральные стачионарные распределения на пространстве $\mathfrak{S}^{\infty}$ параметризуются точками отрезка $[0,1]$. Для эргодической меры $M^{t}$, соответствующей точке $t \in[0,1]$, сумма относительных длин циклов равна $t$ с вероятностью $1, u$ конечномерные распределения этой меры задаются формулой

$$
M_{n}^{t}(h)=\sum_{k=0}^{s} \frac{1}{(n-k) !}\left(\begin{array}{l}
n \\
k
\end{array}\right) t^{n-k}(1-t)^{k}
$$

где $s$ - число неподвижных точек подстановки $h \in \mathfrak{S}_{n}$.

В частности, в классе насыщенных распределений мера Хаара есть единственная стационарная мера.

Не входя в детали, отметим, что с помощью отображения $\Phi$ сдвиг $R_{g}$ можно спроектировать на пространство $\Pi_{\infty}$. Полученная проекция $\bar{R}_{g}$ уже не является взаимно однозначным отображением, а представляет собой марковский оператор (или полиморфизм, см. [2]). Распределение $\bar{\mu} \in \mathscr{M}\left(\Pi_{\infty}\right)$ называется стачионарным, если для любой финитной подстановки $g$ мера $\bar{\mu}$ сохраняется при отображении, являюшемся композицией оператора $\bar{R}_{g}$ и проекции на пространство $\mathscr{M}\left(\Pi_{\infty}\right)$ перестановочных распределений. Теорема 3 , переформулированная в терминах перестановочных разбиений, дает описание всех стационарных распределений на $\Pi_{\infty}$. Мы не будем подробно останавливаться на соответствующих точных формулировках.

Эквивалентная постановка задачи состоит в описании распределений на симплексе $\Sigma$, инвариантных относительно некоторого семейства марковских операторов. Именно, рассмотрим оператор $\widehat{T}_{g}$, который пе- 
реводит точку $x \in \Sigma$ в точку $\phi\left(\omega_{x} g\right)$, где $\omega_{x}$ - центральная последовательность случайных подстановок с распределением $P^{x}$.

Теорема 2. Эргодические меры на симплексе $\Sigma$ для действия семейства операторов $\left\{\widehat{T}_{g}\right\}_{g \in \mathfrak{S}_{\infty}}$ параметризуются точками отрезка $[0,1]$. Эргодическое распределение, соответствующее точке $t \in[0,1]$, сосредоточено на симплексе $\Sigma_{t}$ монотонных последовательностей $c$ суммой $t$ и равно образу меры Пуассона-Дирихле $P D(1)$ под действием гомотетии $x \mapsto t x$.

В частности, на симплексе монотонных последовательностей с единичной суммой мера Пуассона-Дирихле $P D(1)$ есть единственная инвариантная мера. Таким образом, мы получаем новую характеризацию распределения $P D(1)$.

Материал распределен по пунктам следующим образом. Пункт 2 содержит необходимые сведения о пространстве виртуальных подстановок и центральных мерах на нем. В п. 3 приводятся различные формулировки основной теоремы. Доказательство основной теоремы разбито на три части. В первой части (леммы 1,2 ) с помощью эргодического меmода [1] исходная задача сводится к набору условий, формулирующихся В терминах распределений на конечных симметрических группах. В п. 5 доказан основной частный случай теоремы 3 , а именно, единственность стационарного распределения в классе насыщенных мер. Основная идея доказательства состоит в рассмотрении сдвига на случайную подстановку $g \in \mathfrak{S}_{n}$. Эта дополнительная рандомизация обеспечивает хорошие равномерные свойства полученной последовательности подстановок, что позволяет доказать, что ее распределение сколь угодно близко к мере Хаара. Наконец, в п. 6 теорема 3 сводится к этому основному частному случаю.

Автор благодарит А. М. Вершика за постановку задачи, ценные советы и помощь в работе над статьей, а также С. В. Керова и М.И. Гордина за многочисленные полезные обсуждения.

2. Пространство виртуальных подстановок. В этом пункте мы подробно изложим необходимые сведения о пространстве виртуальных подстановок и центральных мерах на нем.

$\mathrm{O}$ п р е д е л е н и е. Для заданного подмножества $J \subset[n]$ и подстановки $w \in \mathfrak{S}_{n}$ обозначим через $\pi_{n, J} w$ подстановку множества $J$, получаемую удалением из циклов подстановки $w$ всех элементов, не принадлежащих $J$. Перестановка $\pi_{n, J} w$ называется производной подстановкой для $w$ на подмножестве $J$.

Производную подстановку на множестве $J=[m]$ обозначим через $\pi_{n, m} w$. Индекс $n$ мы будем обычно опускать, если он определен контекстом. Например, если $w=(6351)(42)(7)$, то $\pi_{4} w=(31)(42)$.

О п р ед ел ени е. Пространством виртуальных подстановок 
$\mathfrak{S}^{\infty}$ называется проективный предел $\mathfrak{S}^{\infty}=\lim _{\longleftarrow} \mathfrak{S}_{n}$ конечных симметрических групп $\mathfrak{S}_{n}$ относительно канонических проекций $\pi_{n+1, n}: \mathfrak{S}_{n+1} \rightarrow \mathfrak{S}_{n}$.

Таким образом, виртуальная подстановка есть последовательность $\omega=\left(w_{1}, w_{2}, \ldots\right) \in \mathfrak{S}_{1} \times \mathfrak{S}_{2} \times \cdots$ такая, что $w_{n}$ - производная подстановка для $w_{n+1}$ при всех $n \in \mathbf{N}$.

Заметим, что проекция $\pi_{n}$ коммутирует со сдвигами на элементы группы $\mathfrak{S}_{n}$, т.е. для любого $N>n$ равенство $\pi_{n}\left(g_{1}^{-1} h g_{2}\right)=g_{1}^{-1} \pi_{n}(h) g_{2}$ выполнено для любого $h \in \mathfrak{S}_{N}$ и произвольных $g_{1}, g_{2} \in \mathfrak{S}_{n}$. Таким образом, корректно определено действие группы $G=\mathfrak{S}_{\infty} \times \mathfrak{S}_{\infty}$ на пространстве виртуальных подстановок $\mathfrak{S}^{\infty}$ по формуле (1).

Последовательность $\left\{\mu_{n}\right\}$ распределений на конечных симметрических группах $\mathfrak{S}_{n}$ называется когерентной, если она согласована относительно проекций $\pi_{n+1, n}$, т.е. $\pi_{n+1, n} \mu_{n+1}=\mu_{n}$ при любом $n \in \mathbf{N}$. Каждая когерентная последовательность распределений задает борелевскую меру $\mu=\lim _{\longleftarrow} \mu_{n}$ на пространстве виртуальных подстановок $\mathfrak{S}^{\infty}$, и каждая борелевская мера на $\mathfrak{S}^{\infty}$ представляется в таком виде. Далее все рассматриваемые меры предполагаются борелевскими вероятностными. Если все меры $\mu_{n}$ центральны, т.е. инвариантны относительно внутренних автоморфизмов $\mathfrak{S}_{n}$, то предельное распределение $\mu$ инвариантно относительно действия диагональной подгруппы $K=\left\{\left(g_{1}, g_{2}\right) \in G: g_{1}=\right.$ $\left.g_{2}\right\}$. Меры на пространстве виртуальных подстановок $\mathfrak{S}^{\infty}$, инвариантные относительно подгруппы $K$, называются чентральными (они соответствуют центральным мерам графа ветвления классов сопряженности симметрических групп, см. [3], [5]). Обозначим через $\mathscr{M}^{K}\left(\mathfrak{S}^{\infty}\right)$ множество всех центральных распределений.

Теорема $1^{\prime}$ дает полное описание центральных распределений на пространстве виртуальных подстановок. В частности, из этой теоремы следует, что для каждой борелевской меры $\nu$ на симплексе $\Sigma$ существует и единственна центральная мера $\mu$ на $\mathfrak{S}^{\infty}$, для которой $\nu$ есть распределение вектора относительных длин циклов. Пусть $\mathscr{M}(\Sigma)-$ множество борелевских распределений на симплексе $\Sigma$. Обозначим через $\rho$ оператор, сопоставляющий распределению $\nu \in \mathcal{M}(\Sigma)$ соответствующую центральную меру $\mu=\int P^{x} d \nu(x)$.

П р и м е р 2. Простейшим описанием меры Пуассона-Дирихле $P D(1)$, которая является распределением длин циклов виртуальных подстановок относительно меры Хаара (см. пример 1), является следующий прочесс ломания палки. Пусть $U_{1}, U_{2}, \ldots$ - последовательность независимых случайных величин, равномерно распределенных на отрезке [0,1]. Положим $V_{n}=U_{n} \prod_{i=1}^{n-1}\left(1-U_{i}\right)$. Мера Пуассона-Дирихле $P D(1)$ есть распределение порядковых статистик $V_{(1)} \geqslant V_{(2)} \geqslant \cdots$ последовательности $V_{1}, V_{2}, \ldots$

Опишем теперь модификацию конструкции Кингмана для построе- 
ния эргодических перестановочных разбиений, с помощью которой можно построить случайную виртуальную подстановку с распределением $P^{x}$. Обозначим $x_{0}=1-x_{1}-x_{2}-\cdots$. Будем размещать элементы $1,2, \ldots$ последовательно в циклы и приписывать этим циклам случайные метки, причем на каждом шаге новый элемент с вероятностью $x_{0}$ будет образовывать особый цикл длины 1, который не имеет метки и к которому запрещено добавлять новые элементы. Итак,

(1) на первом шаге элемент 1 с вероятностью $x_{0}$ образует особый цикл и с вероятностью $x_{j}(j=1,2, \ldots)$ - неособый цикл длины 1 с меткой $j$;

(2) если элементы $1, \ldots, m$ уже размешены и образуют несколько особых циклов и $k$ неособых циклов с метками $i_{1}, \ldots, i_{k}$, то элемент $m+1$ с вероятностью $x_{i,}(j=1, \ldots, k)$ помещается на одну из возможных позиций в $j$-м неособом цикле, с вероятностью $x_{i}\left(i \neq i_{1}, \ldots, i_{k}\right)$ образует новый неособый цикл длины 1 с меткой $i$ и с вероятностью $x_{0}$ образует новый особый цикл.

После $n$-го шага этой процедуры мы получаем случайную подстановку $w_{n} \in \mathfrak{S}_{n}$. Из конструкции следует, что последовательность $\left\{w_{n}\right\}$ когерентна. Нетрудно убедиться, что ее распределение есть $P^{x}$. В частности, при $x=(0,0, \ldots)$ распределение $P^{0}$ сосредоточено на последовательности, состоящей из тождественных подстановок.

П р и м е р 3. Проекция эргодической меры $P^{x}$ на симметрическую группу $\mathfrak{S}_{2}$ задается формулой

$$
P_{2}^{x}((1)(2))=2 \sum_{1 \leqslant i<j<\infty} x_{i} x_{j}+x_{0}^{2}+2 x_{0}\left(1-x_{0}\right), \quad P_{2}^{x}((12))=\sum_{i=1}^{\infty} x_{i}^{2} .
$$

Заметим, что сумма относительных длин циклов виртуальной подстановки не обязательно равна единице. Простейшим примером является виртуальная подстановка $e=\left(e_{1}, e_{2}, \ldots\right)$, где $e_{i} \in \mathfrak{S}_{i}$ - тождественная подстановка. Ясно, что длины всех ее циклов равны нулю.

О п р е д е л е н и е. Щентральное распределение $\mu$ на пространстве виртуальных подстановок называется насыщенныл, если сумма относительных длин циклов равна единице почти наверное относительно $\mu$. Когерентное семейство $\left\{\mu_{n}\right\}$ центральных распределений на симметрических группах называется насыщеннылм, если предельная мера $\mu=\lim \mu_{n}$ на пространстве виртуальных подстановок является насыщенной.

Задача об общих центральных распределениях на пространстве виртуальных подстановок в большинстве случаев может быть сведена к задаче для насыщенных распределений. Поясним это подробнее.

Пусть $t \in[0,1]$. Обозначим через $\Sigma_{t}=\left\{x \in \Sigma: \sum_{i=1}^{\infty} x_{i}=t\right\}$ симплекс монотонных последовательностей с суммой $t$. При $t \neq 0$ стандартный симплекс $\Sigma_{1}$ отождествляется с $\Sigma_{t}$ при помошц гомотетии $\Gamma_{t}: x \mapsto x t$. Через $\Gamma_{0}$ обозначим постоянное отображение из $\Sigma_{1}$ в 
$\Sigma_{0}=\{0\}$. Пусть $\nu$ - произвольное распределение на симплексе $\Sigma$. Обозначим через $\tau$ образ меры $\nu$ при отображении $x \mapsto x_{1}+x_{2}+\cdots$, и через $\nu^{t}$ - условное распределение $\nu$ на симплексе $\Sigma_{t}$, так что $\nu=\int_{0}^{1} \nu^{t} d \tau(t)$. Пусть $\bar{\nu}^{t}=\Gamma_{t}^{-1} \nu^{t}$. Нетрудно показать, что конечномерные распределения центральной меры $\mu$, соответствующей $\nu$, задаются формулой

$$
\mu_{n}(h)=\int_{0}^{1} \sum_{k=0}^{s}\left(\begin{array}{l}
n \\
k
\end{array}\right) t^{n-k}(1-t)^{k} \mu_{n-k}^{t}\left(h^{k}\right) d \tau(t)
$$

где $\mu^{t}=\int_{\Sigma_{1}} P^{y} d \bar{\nu}^{t}(y)$ - насышенное центральное распределение, соответствующее $\bar{\nu}^{t}, s-$ число неподвижных точек подстановки $h \in \mathfrak{S}_{n}$ и $h^{k}$ - подстановка, полученная из $h$ удалением $k$ неподвижных точек.

3 а м е ч а н и е. Пространство виртуальных подстановок $\mathfrak{S}^{\infty}$ было введено в статье [11]. В этой работе указано семейство квазиинвариантных распределений на пространстве $\mathfrak{S}^{\infty}$ относительно описанного действия группы $G=\mathfrak{S}_{\infty} \times \mathfrak{S}_{\infty}$ и изучено связанное с ним семейство унитарных представлений бесконечной симметрической группы. В серии последующих работ [6]-[8] рассмотрены различные свойства пространства виртуальных подстановок.

3. Основная теорема. Бесконечная симметрическая группа $\mathfrak{S}_{\infty}$ действует на пространстве виртуальных подстановок двусторонними сдвигами (1). Обозначим через $R_{g}: \mathfrak{S}^{\infty} \rightarrow \mathfrak{S}^{\infty}$ правый сдвиг на подстановку $g \in \mathfrak{S}_{\infty}$.

Нетрудно показать, что образ $\mu^{g}=R_{g} \mu$ центрального распределения $\mu$ обладает тем свойством, что относительные длины циклов (2) существуют для почти всех относительно $\mu^{g}$ виртуальных подстановок. Поэтому можно определить проекцию сдвига $R_{g}$ на симплекс $\Sigma$, которая будет являться уже марковским оператором. Этот оператор переводит точку $x \in \Sigma$ в точку $\phi\left(\omega_{x} g\right)$, где $\omega_{x}$ - случайная виртуальная подстановка с распределением $P^{x}$. Таким образом, соответствующий оператор $\widehat{T}_{g}$ замыкает коммутативную диаграмму

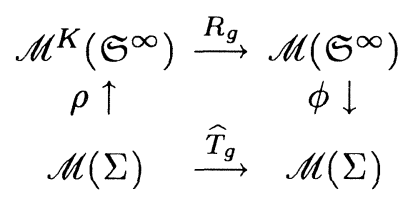

Нетрудно проверить, что оператор $\widehat{T}_{g}$ зависит только от класса сопряженности подстановки $g$, т.е. от ее цикловой структуры.

II р и м е р 4. Очевидно, что $\widehat{T}_{e}-$ тождественный оператор. Простейшим нетривиальным примером является оператор, соответствующий транспозиции. Из конструкции эргодической меры $P^{x}$ следует, что ограничение $T_{(1,2)}$ оператора $\widehat{T}_{(1,2)}$ на симплекс $\Sigma_{1}$ задается следующей фор- 
мулой. Обозначим через $V$ оператор, переставляющий координаты последовательности в невозрастающем порядке. Тогда

$$
T_{(1,2)} x=\left\{\begin{array}{c}
V\left(x_{i}+x_{j}, x_{1}, \ldots, x_{i-1}, x_{\imath+1}, \ldots, x_{j-1}, x_{j+1}, \ldots\right), \quad i<j, \\
\text { с вероятностью } 2 x_{\imath} x_{j} \\
V\left(t, x_{i}-t, x_{1}, \ldots, x_{i-1}, x_{i+1}, \ldots\right), \quad i=1,2, \ldots, \\
\text { с вероятностью } d t, t \in\left[0, x_{i}\right] .
\end{array}\right.
$$

Ясно, что если мера $\mu$ инвариантна относительно сдвига $R_{g}$, то распределение $\nu=\phi \mu$ инвариантно относительно оператора $\widehat{T}_{g}$. Следовательно, при любом $g \in \mathfrak{S}_{\infty}$ распределение Пуассона-Дирихле $P D(1)$ $\widehat{T}_{g}$-инвариантно.

Нетрудно видеть, что множество $\Sigma_{t}=\left\{x \in \Sigma: \sum x_{i}=t\right\}$ инвариантно относительно $\widehat{T}_{g}$ при любом $t \in[0,1]$ (поскольку сдвиг на финитную подстановку не меняет сумму относительных длин циклов виртуальной подстановки). Оказывается, что эти множества являются эргодическими компонентами, и эргодическая мера, сосредоточенная на $\Sigma_{t}$, есть образ распределения Пуассона-Дирихле $P D(1)$ под действием гомотетии $\Gamma_{t}: \Sigma_{1} \rightarrow \Sigma_{t}$

Теорема 2. Эргодические меры на симплексе $\Sigma$ для действия семейства операторов $\left\{\widehat{T}_{g}\right\}_{g \in \mathfrak{S}_{\infty}}$ параметризуются точками отрезка $[0,1]$. Эргодическое распределение, соответствующее точке $t \in[0,1]$, сосредоточено на симплексе $\Sigma_{t}$ и равно $P D_{t}(1)=\Gamma_{t}(P D(1))$.

Следствие. Общий вид распределения на симплексе $\Sigma$, инвариантного относительно семейства операторов $\left\{\widehat{T}_{g}\right\}_{g \in \mathfrak{S}_{\infty}}$, задается формулой

$$
\nu=\int_{0}^{1} P D_{t}(1) d \tau(t),
$$

где $\tau$ - произвольное борелевское распределение на отрезке $[0,1]$.

Описанная задача допускает другую формулировку. Пусть $\nu \in$ $\mathscr{M}(\Sigma)$. Рассмотрим щентральную меру $\rho\left(\widehat{T}_{g} \nu\right)$, соответствующую распределению $\widehat{T}_{g} \nu$. По определению оператора $\widehat{T}_{g}$, имеем $\widehat{T}_{g} \nu=\phi \mu^{g}$, где $\mu=\rho \nu$ - центральная мера на $\mathfrak{S}^{\infty}$, соответствующая $\nu$, и $\mu^{g}=R_{g} \mu$. Следовательно, $\rho\left(\widehat{T}_{g} \nu\right)=\int_{\Sigma} P^{x} d\left(\phi \mu^{g}\right)(x)$. Итак, распределение $\nu$ инвариантно относительно $\widehat{T}_{g}$ тогда и только тогда, когда распределение $\mu$ удовлетворяет условию

$$
\mu=\int_{\Sigma} P^{x} d\left(\phi \mu^{g}\right)(x)
$$

Центральную меру $\mu$ на пространстве виртуальных подстановок будем называть стачионарной, если она удовлетворяет условию (5) для Bcex $g \in \mathfrak{S}_{\infty}$. 
Правая часть формулы (5) определяет проекцию $\mathbb{P} \mu^{g}$ меры $\mu^{g}$ на пространство $M^{K}\left(\mathfrak{S}^{\infty}\right)$ центральных (т.е. $K$-инвариантных) мер, а именно, $P \mu^{g}$ есть единственная центральная мера, имеющая то же распределение относительных длин циклов, что и $\mu^{g}$. Заметим, что группа $K$, изоморфная $\mathfrak{S}_{\infty}$, локально конечна. А. М. Вершик предложил использовать эргодический метод [1] для определения проекции $\mathbb{P}$.

Лемма 1. Пусть $\eta$ - распределение на пространстве виртуальных подстановок, такое что относительные длины чиклов существуют почти наверное относительно $\eta$. Тогда проекиия распределения $\eta$ на пространство чентральных мер $M^{K}\left(\mathfrak{S}^{\infty}\right)$ задается формулой

$$
P \eta=\lim _{N \rightarrow \infty} \frac{1}{N !} \sum_{u \in \mathfrak{S}_{N}} u^{-1} \eta u .
$$

3ам е ч а н ие. Здесь и далее имеется в виду слабая сходимость распределений.

Доказ а т е л ь с т в о. Обозначим $\eta^{N}=(N !)^{-1} \sum_{u \in \mathfrak{S}_{N}} u^{-1} \eta u$. Пространство $\mathscr{M}\left(\mathfrak{S}^{\infty}\right)$ компактно, поэтому из последовательности $\left\{\eta^{N}\right\}$ можно выбрать слабо сходящуюся подпоследовательность $\left\{\eta^{N_{k}}\right\}$. Пусть $\eta_{0}$ - предел этой подпоследовательности. Поскольку сопряжение не меняет длин циклов, то $\phi\left(u^{-1} \eta u\right)=\phi(\eta)$ для любого $u \in \mathfrak{S}_{\infty}$, следовательно, $\phi\left(\eta_{0}\right)=\phi(\eta)$.

Обозначим $K_{n}=K \cap\left(\mathfrak{S}_{n} \times \mathfrak{S}_{n}\right)$, так что $K=\cup K_{n}$. Очевидно, что мера $\eta^{N}$ инвариантна относительно $K_{n}$ при $N \geqslant n$. Отсюда следует, что мера $\eta_{0}$ инвариантна относительно $K_{n}$ при любом $n \in \mathbb{N}$, т.е. $\eta_{0} \in$ $\mathscr{M}^{K}\left(\mathfrak{S}^{\infty}\right)$.

Итак, $\eta_{0}$ есть центральная мера, имеющая то же распределение длин циклов, что и $\eta$, т.е. $\eta_{0}=P \eta$. Таким образом, каждая сходящаяся подпоследовательность последовательности $\left\{\eta^{N}\right\}$ сходится к $P \eta$, откуда следует (6).

3 а м е ч а н и е. Таким образом, определение стационарной меры можно записать в виде

$$
\mu=\lim _{N \rightarrow \infty} \frac{1}{N !} \sum_{u \in \mathfrak{S}_{N}} u^{-1} \mu^{g} u, \quad g \in \mathfrak{S}_{\infty} .
$$

Напомним, что обычное понятие стационарного распределения для группового действия определено в случае действия локально компактной группы следующим образом (см. [10]). Пусть $\mathfrak{G}$ - локально компактная группа, действующая на измеримом пространстве $X$, и $\varkappa$ - распределение на $\mathfrak{G}$. Распределение $\eta$ на пространстве $X$ называется стационарным (относительно $\varkappa$ ), если оно удовлетворяет условию $\eta=\int_{\mathfrak{G}} \eta^{a} d \varkappa(a)$, где $\eta^{a}$ есть образ меры $\eta$ под действием элемента $a \in \mathfrak{G}$. Формула (7) является естественным обобщением этого определения для действия локально конечной группы. 
Ввиду формулы (4) теорема 2 эквивалентна следующей теореме 3.

Теорема 3. Общий вид стационарной вероятностной меры $\mu=$ $\lim _{\longleftarrow} \mu_{n}$ на пространстве виртуальных подстановок задается формулой

$$
\mu_{n}(h)=\sum_{k=0}^{s} \frac{1}{(n-k) !}\left(\begin{array}{l}
n \\
k
\end{array}\right) \int_{0}^{1} t^{n-k}(1-t)^{k} d \tau(t),
$$

где $s-$ число неподвижных точек подстановки $h \in \mathfrak{S}_{n} u \tau-$ произвольное борелевское распределение на отрезке $[0,1]$.

Среди насыщенных распределений (соответствуюших мере $\tau$, сосредоточенной в точке $t=1$ ) единственной стационарной мерой является мера Хаара. Таким образом, в классе насыщенных распределений нет неинвариантных стационарных мер.

3 а м е ч а н и е. Поскольку $\left\{\widehat{T}_{g}\right\}$ не является групповым действием $\mathfrak{S}_{\infty}$, естественно задать вопрос об описании инвариантных распределений относительно одного оператора $\widehat{T}_{(1,2)}$, соответствующего транспозициям. Этот вопрос тем более интересен, что оператор $\widehat{T}_{(1,2)}$ имеет очень простую форму (см. пример 4). Гипотеза состоит в том, что для одного оператора $\widehat{T}_{(1,2)}$ запас инвариантных распределений такой же, как и для всего семейства $\left\{\widehat{T}_{g}\right\}_{g \in \mathfrak{S}_{\infty}}$, однако этот вопрос пока остается открытым.

4. Сведение к конечномерным условиям. Первая часть доказательства основной теоремы состоит в сведении исходной бесконечномерной задачи к (бесконечному) набору условий, формулирующихся в терминах распределений на конечных симметрических группах.

Лемма 2. Пусть $\mu$ - чентральное распределение на пространстве виртуальных подстановок, $\left\{\mu_{n}\right\}$ - соответствующее когерентное семейство распределений на симметрических группах, $g \in \mathfrak{S}_{k}$. Тогда конечномерные проекции распределения $P \mu^{g}$ задаются формулой

$$
\left(P \mu^{g}\right)_{n}(u)=\sum_{\substack{w \in \mathfrak{S}_{n+k} \\ \pi_{n}(w)=u}} \mu_{n+k}\left(w g^{n}\right), \quad u \in \mathfrak{S}_{n},
$$

где $g^{n} \in \mathfrak{S}_{n+k}$ - подстановка такая, что $g^{n}(i)=i$ при $i=1, \ldots, n, u$ $\pi_{\{n+1, \ldots, n+k\}} g^{n}$ имеет ту же иикловую структуру, что и $g$.

Д о к аз а т ель с тв о. Пусть $\eta=P \mu^{g}$ и $u \in \mathfrak{S}_{n}$. Тогда

$$
\eta_{n}(u)=\lim _{N \rightarrow \infty} \frac{1}{N !} \sum_{h \in \mathfrak{S}_{N}} \sum_{\substack{w \in \mathfrak{S}_{N} \\ \pi_{n}(w)=u}} \mu_{N}\left(h^{-1} w h g\right) .
$$

Обозначим через $\Pi_{n}$ множество всех разбиений числа $n$. Классы сопряженности симметрической группы $\mathfrak{S}_{n}$ параметризуются элементами $\lambda \in \Pi_{n}$. Таким образом, существует взаимно однозначное соответствие 
между центральными мерами на $\mathfrak{S}_{n}$ и распределениями на $\Pi_{n}$. Обозначим через $[\lambda]$ класс сопряженности, соответствующий $\lambda \in \Pi_{n}$, т.е. множество подстановок, длины циклов которых образуют разбиение $\lambda$, и через $|\lambda|$ - мощность этого класса. Мере $\eta$ соответствует распределение

$$
\bar{\eta}_{n}(\lambda)=\sum_{v \in[\lambda]} \eta_{n}(v)=|\lambda| \eta_{n}(u)
$$

где $u$ - произвольный представитель класса $[\lambda]$. Таким образом,

$$
\bar{\eta}_{n}(\lambda)=\lim _{N \rightarrow \infty} \frac{1}{N !} \sum_{h \in \mathfrak{S}_{N}} \sum_{\substack{w \in \mathfrak{S}_{N} \\ \pi_{n}(w) \in[\lambda]}} \mu_{N}\left(h^{-1} w h g\right)
$$

Обозначим допредельное выражение через $\sigma_{N}$. Заметим, что $\sigma_{N}$ можно записать в виде

$$
\sum_{v \in \mathfrak{S}_{N}} \mu_{N}(v) \frac{1}{N !} \#\left\{h \in \mathfrak{S}_{N}: \pi_{n}\left(h v g^{-1} h^{-1}\right) \in[\lambda]\right\}=\sum_{v \in \mathfrak{S}_{N}} \mu_{N}(v) q(v, \lambda)
$$

где $q(v, \lambda)$ - переходные вероятности марковского оператора, действующего из $\mathfrak{S}_{N}$ в $\Pi_{n}$ и переводящего подстановку $v \in \mathfrak{S}_{N}$ в разбиение, соответствующее классу подстановки $\pi_{n}(w)$, где $w$ - случайная подстановка, равномерно распределенная на классе, содержащем $v g^{-1}$. Заметим, что рассмотрение производной подстановки на множестве $[n]$ случайного представителя класса, содержащего $v g^{-1}$, равносильно рассмотрению производной подстановки $v g^{-1}$ на случайном подмножестве $J \subset[N]$ мощности $n$, т.е.

$$
q(v, \lambda)=\frac{1}{\left(\begin{array}{l}
N \\
n
\end{array}\right)} \#\left\{J \subset[N]: \# J=n, \pi_{J}\left(v g^{-1}\right) \in[\lambda]\right\} .
$$

Обозначим через $\delta_{\lambda}$ характеристическую функцию класса $[\lambda]$. Тогда

$$
\sum_{v \in \mathfrak{S}_{N}} \mu_{N}(v) q(v, \lambda)=\frac{1}{\left(\begin{array}{l}
N \\
n
\end{array}\right)} \sum_{\substack{J \subset[N] \\
\# J=n}} \sum_{v \in \mathfrak{S}_{N}} \mu_{N}(v) \delta_{\lambda}\left(\pi_{J}\left(v g^{-1}\right)\right)
$$

Пусть $N \geqslant n+k$. Поскольку $P \mu^{g}$ зависит только от цикловой структуры подстановки $g$, можно считать, что $g \in \mathfrak{S}[n+1, \ldots, n+k]$. Пусть $J=$ $\left\{j_{1}, \ldots, j_{n}\right\}$. Предположим, что

$$
\left\{j_{1}, \ldots, j_{n}\right\} \cap\{n+1, \ldots, n+k\}=\varnothing .
$$

Вероятность того, что случайное подмножество мощности $n$ множества $[N]$ обладает этим свойством, равна $\left(\begin{array}{c}N-k \\ n\end{array}\right) /\left(\begin{array}{c}N \\ n\end{array}\right)$. Рассмотрим подстановку $h \in \mathfrak{S}_{N}$ такую, что

$$
h\left(j_{1}\right)=1, \ldots, h\left(j_{n}\right)=n \quad \text { и } \quad h(i)=i
$$


при $i \notin J$. Очевидно, что $h$ коммутирует с $g$. В силу центральности меры $\mu_{N}$ отсюда следует, что сумма

$$
\begin{aligned}
& \sum_{v \in \mathfrak{S}_{N}} \mu_{N}(v) \delta_{\lambda}\left(\pi_{J}\left(v g^{-1}\right)\right)=\sum_{v \in \mathfrak{S}_{N}} \mu_{N}(v) \delta_{\lambda}\left(\pi_{J}\left(h v h^{-1} g^{-1}\right)\right) \\
& =\sum_{v \in \mathfrak{S}_{N}} \mu_{N}(v) \delta_{\lambda}\left(\pi_{J}\left(h v g^{-1} h^{-1}\right)\right)=\sum_{v \in \mathfrak{S}_{N}} \mu_{N}(v) \delta_{\lambda}\left(\pi_{n}\left(v g^{-1}\right)\right)
\end{aligned}
$$

не зависит от выбора подмножества $J$, если оно удовлетворяет условию (8). Кроме того, поскольку $g \in \mathfrak{S}[n+1, \ldots, n+k]$, последняя сумма равна $\sum_{v \in \mathfrak{S}_{n+k}} \mu_{n+k}(v) \delta_{\lambda}\left(\pi_{n}\left(v g^{-1}\right)\right)$. Следовательно,

$$
\sigma_{N}=\frac{\left(\begin{array}{c}
N-k \\
n
\end{array}\right)}{\left(\begin{array}{c}
N \\
n
\end{array}\right)} \sum_{v \in \mathfrak{S}_{n+k}} \mu_{n+k}(v) \delta_{\lambda}\left(\pi_{n}\left(v g^{-1}\right)\right)+R_{N}
$$

где $\left|R_{N}\right| \leqslant 1-\left(\begin{array}{c}N-k \\ n\end{array}\right) /\left(\begin{array}{l}N \\ n\end{array}\right) \rightarrow 0$ при $N \rightarrow \infty$, откуда следует утверждение леммы.

5. Основная лемма. Как было отмечено выше, утверждения об общих центральных мерах на пространстве виртуальных подстановок обычно сводятся х случаю насыщенных мер. В соответствии с этим принципом, мы докажем сначала, что в классе насыщенных распределений мера Хаара есть единственная стационарная мера. Точнее, имеет место даже более сильное утверждение. Приведем две эквивалентные формулировки.

Теорема 4. Пусть $T_{g}$ - ограничение оператора $\widehat{T}_{g}$ на инвариантное подмножество $\Sigma_{1}$. Обозначим через $T_{n}(n=1,2, \ldots)$ усреднение операторов $T_{g}$ по симметрической гpynпе $\mathfrak{S}_{n}$ :

$$
T_{n}=\frac{1}{n !} \sum_{g \in \mathfrak{S}_{n}} T_{g}
$$

Мера Пуассона-Дирихле $P D(1)$ есть единственное распределение на симплексе $\Sigma_{1}$, инвариантное относительно $\left\{T_{n}\right\}_{n \in \mathbb{N}}$.

Теорема 5. Пусть $R_{n}$ - марковский оператор сдвига на случайную подстановку, равномерно распределенную на группе $\mathfrak{S}_{n}$. Мера Хаара на пространстве виртуальных подстановок есть единственная иентральная мера, удовлетворяюшая условию $\mu=P\left(R_{n} \mu\right), n \in \mathbb{N}$.

В силу леммы 2 теоремы 4 и 5 следуют из основной леммы 3 . Для фиксированных $n, k \in \mathbb{N}$ обозначим $\mathfrak{S}^{k}=\mathfrak{S}[n+1, \ldots, n+k]$.

Основная лемма 3. Пусть $\left\{\mu_{n}\right\}$ - насыщенное когерентное семейство чентральных распределений на симметрических группах $\mathfrak{S}_{n}$, удовлетворяюшее условиям

$$
\sum_{\substack{w \in \mathfrak{S}_{n+k} \\ \pi_{n}(w)=u}} \frac{1}{k !} \sum_{g \in \mathfrak{S}^{k}} \mu_{n+k}(w g)=\mu_{n}(u) \quad \forall n, k \in \mathbf{N}, \forall u \in \mathfrak{S}_{n} .
$$


Тогда распределение $\mu_{n}$ совпадает с мерой Хаара $m_{n}$ на $\mathfrak{S}_{n}$ при любом $n \in \mathbf{N}$.

Д о к а з а т е л в с т в о. Заметим, что условие (9) можно переписать в виде $\mu_{n}(u)=\sum_{w \in \mathfrak{S}_{n+k}} \mu_{n+k}(w) P(w, u)$, где $P(w, u)$ - переходные вероятности марковского оператора, действующего из $\mathfrak{S}_{n+k}$ в $\mathfrak{S}_{n}$ и переводящего подстановку $w \in \mathfrak{S}_{n+k}$ в подстановку $\pi_{n}(w g)$, где $g$ равномерно распределена на $\mathfrak{S}^{k}$. Переходя к соответствуюшему распределению $\bar{\mu}_{n+k}$ на пространстве разбиений $\Pi_{n+k}$, получаем

$$
\mu_{n}(u)=\sum_{w \in \mathfrak{S}_{n+k}} \mu_{n+k}(w) P(w, u)=\sum_{\lambda \in \Pi_{n+k}} \bar{\mu}_{n+k}(\lambda) \bar{P}(\lambda, u),
$$

где $\bar{P}(\lambda, u)=(|\lambda|)^{-1} \sum_{w \in[\lambda]} P(w, u)$.

Следующая лемма показывает, что если $n$ фиксировано, $N=n+k$ достаточно велико и разбиение $\lambda \in \Pi_{N}$ содержит мало единичных слагаемых, то переходная вероятность $\bar{P}(\lambda, u)$ близка к мере Хаара $m_{n}(u)=1 / n$ !

Лемма 4. Пусть $\lambda \in \Pi_{N}$. Обозначим через $b(\lambda)$ число единичных слагаемых в разбиении $\lambda$. Для любой подстановки $u \in \mathfrak{S}_{n}$

$$
\bar{P}(\lambda, u)=m_{n}(u)+R(\lambda, u),
$$

где

$$
|R(\lambda, u)| \leqslant 1-\frac{(N-b(\lambda))(N-b(\lambda)-2) \cdots(N-b(\lambda)-2(n-1))}{N(N-1) \cdots(N-(n-1))} .
$$

Д ок а з а те л в с тво. Будем называть младиими элементы $1, \ldots, n$ множества $[N]$ и стариими - оставшиеся элементы $n+1, \ldots, N$. Рассмотрим множество $D$ всех подстановок из $\mathfrak{S}_{N}$ таких, что прообразы младших элементов являются старшими:

$$
D=\left\{w \in \mathfrak{S}_{N}: w^{-1}(i) \in\{n+1, \ldots, n+k\}, i=1, \ldots, n\right\} .
$$

Пусть $w \in D$. Обозначим $j_{1}=w^{-1}(1), \ldots, j_{n}=w^{-1}(n)$. Зафиксируем изоморфизм групп $\mathfrak{S}_{n}$ и $\mathfrak{S}\left[j_{1}, \ldots, j_{n}\right]$, отождествляющий $i$ и $j_{i}$. Очевидно, что он отождествляет $\pi_{n}(w) \mathrm{c} \pi_{\left\{j_{1}, \ldots, j_{n}\right\}}(w)$. Более того, для любой подстановки $g \in \mathfrak{S}^{k}$ имеет место равенство $(w g)^{-1}(i)=w^{-1}(i)$ при всех $i=1, \ldots, n$, поскольку младшие элементы $1, \ldots, n$ являются неподвижными точками подстановки $g$. Поэтому $\pi_{n}(w g)$ отождествляется с $\pi_{\left\{j_{1}, \ldots, j_{n}\right\}}(w g)$ при всех $g \in \mathfrak{S}^{k}$. Таким образом, распределение производной подстановки $\pi_{n}(w g)$ на группе $\mathfrak{S}_{n}$ совпадает в смысле выбранного изоморфизма с распределением подстановки $\pi_{\left\{j_{1}, \ldots, j_{n}\right\}}(w g)$ на $\mathfrak{S}\left[j_{1}, \ldots, j_{n}\right]$. Но очевидно, что если подстановка $g$ равномерно распределена на $\mathfrak{S}^{k}$, то подстановка $\pi_{\left\{j_{1}, \ldots, j_{n}\right\}}(w g)$ равномерно распределена на $\mathfrak{S}\left[j_{1}, \ldots, j_{n}\right]$. 
Мы показали, что $P(w, u)=m_{n}(u)$ при $w \in D$. Отсюда

$$
\bar{P}(\lambda, u)=\frac{1}{|\lambda|} \sum_{w \in[\lambda]} P(w, u)=\frac{\#(D \cap|\lambda|)}{|\lambda|} m_{n}(u)+r(\lambda, u),
$$

где $r(\lambda, u)=(|\lambda|)^{-1} \sum_{w \in[\lambda] \backslash D} P(w, u) \leqslant 1-\#(D \cap|\lambda|) /|\lambda|$. Следовательно,

$$
|R(\lambda, u)| \leqslant\left|r(\lambda, u)-m_{n}(u)\left(1-\frac{\#(D \cap|\lambda|)}{|\lambda|}\right)\right| \leqslant 1-\frac{\#(D \cap|\lambda|)}{|\lambda|} .
$$

Таким образом, нам осталось оценить вероятность того, что случайная подстановка $w \in[\lambda]$ принадлежит множеству $D$.

Для получения равномерно распределенной подстановки $w \in[\lambda]$ мы применяем следующую процедуру. Рассмотрим диаграмму Юнга разбиения $\lambda$ и будем размещать элементы $1, \ldots, n$ последовательно в клетках этой диаграммы, полагая на каждом шаге, что все пустые (незаполненные) клетки имеют равные вероятности. Строчки заполненной диаграммы интерпретируем как циклы. Таким образом, мы получаем случайную подстановку $w \in[\lambda]$. Очевидно, что она равномерно распределена на классе $[\lambda]$.

На первом шаге процедуры назовем плохими клетки диаграммы, составляющие строки длины 1 . Напомним, что количество таких клеток обозначалось $b(\lambda)$, так что вероятность того, что элемент 1 попадет в хорошую (т.е. не плохую) клетку, есть $(N-b(\lambda)) / N$. После того, как элемент 1 размещен, назовем плохой клетку, расположенную непосредственно слева от 1 в циклическом порядке. Таким образом, на втором шаге имеется $(N-1)$ свободная клетка и не более $(b(\lambda)+1)$ плохих, так что вероятность того, что и 1 , и 2 попадут в хорошие клетки, по крайней мepe

$$
\frac{N-b(\lambda)}{N} \cdot \frac{N-b(\lambda)-2}{N-1} .
$$

Продолжим процедуру, после каждого шага добавляя к плохим клеткам левого соседа только что занятой клетки. Поскольку каждый раз число плохих клеток увеличивается не более чем на единицу, то вероятность того, что все элементы $1, \ldots, n$ попадут в хорошие клетки, по крайней мepe

$$
\frac{N-b(\lambda)}{N} \cdot \frac{N-b(\lambda)-2}{N-1} \cdots \frac{N-b(\lambda)-2(n-1)}{N-(n-1)} .
$$

Но в этом случае, во-первых, элементы $1, \ldots, n$ не лежат в строках длины 1 (так как клетки 1-строк заведомо плохие) и, во-вторых, прообразы этих элементов лежат в множестве $\{n+1, \ldots, n+k\}$ (так как клетки, находящиеся слева по циклу от клеток, содержащих младшие элементы, 
являются плохими, и, следовательно, заняты старшими элементами), т.е. соответствующая подстановка лежит в множестве $D$. Отсюда

$$
\frac{\#(D \cap|\lambda|)}{|\lambda|} \geqslant \frac{N-b(\lambda)}{N} \cdot \frac{N-b(\lambda)-2}{N-1} \ldots . \frac{N-b(\lambda)-2(n-1)}{N-(n-1)}
$$

что и требовалось доказать.

Чтобы воспользоваться оценкой леммы 4, нужно показать, что в случае насыщенных мер вероятность того, что случайная подстановка имеет много неподвижных точек, асимптотически равна нулю.

Лемма 5. Обозначим $A_{N}(\delta)=\left\{\lambda \in \Pi_{N}: b(\lambda) / N<\delta\right\}, 0<\delta<1$. Если $\left\{\mu_{N}\right\}$ - насыщенное когерентное семейство чентральных распределений на симметрических группах, то для любого $\delta \in(0,1)$

$$
\bar{\mu}_{N}\left(A_{N}(\delta)\right) \rightarrow 1 \quad \text { npu } N \rightarrow \infty
$$

Д ок а з а тел в с т в о. Зафиксируем $\delta \in(0,1)$ и обозначим $A_{n}=$ $A_{n}(\delta)$. Рассмотрим вектор $\phi_{N}(w)=\left(l_{1}(w) / N, l_{2}(w) / N, \ldots\right) \in \Sigma$ нормированных длин циклов подстановки $w \in \mathfrak{S}_{N}$ в невозрастающем порядке. Пусть $\nu_{N}$ - образ меры $\mu_{N}$ под действием отображения $\phi_{N}$. Тогда последовательность мер $\nu_{N}$ слабо сходится к распределению $\nu$ вектора относительных длин циклов виртуальных подстановок относительно предельной меры $\mu=\lim \mu_{N}$. По определению насыщенных мер, распределение $\nu$ сосредоточено на симплексе $\Sigma_{1}$ последовательностей с единичной суммой.

Обозначим через $F_{N}: \Sigma \rightarrow[0,1]$ функцию $F_{N}(x)=\sum_{i: x_{\imath} \geqslant 2 / N} x_{\imath}$. Ясно, что $F_{N}(x) \nearrow \sum_{i=1}^{\infty} x_{i}$ при $N \rightarrow \infty$, так что $F_{N}(x) \nearrow 1$ почти наверное относительно меры $\nu$. Пусть $B_{N}=\left\{x \in \Sigma: F_{N}(x) \geqslant 1-\delta\right\}$. Поскольку $B_{N} \nearrow B=\left\{x: \sum x_{i} \geqslant 1-\delta\right\}$, то $\nu B_{N} \nearrow 1$.

Нетрудно видеть, что $\lambda \in A_{N}$ тогда и только тогда, когда $\phi_{N}(w) \in$ $B_{N}$ для любой подстановки $w \in[\lambda]$, откуда $\bar{\mu}_{N} A_{N}=\nu_{N} B_{N}$. Но поскольку распределения $\nu_{N}$ слабо сходятся к мере $\nu$, последовательность множеств $B_{N}$ возрастает и $\nu B_{N} \nearrow 1$, то мы получаем, что $\nu_{N} B_{N} \rightarrow 1$, откуда следует утверждение леммы.

Теперь мы можем завершить доказательство основной леммы 3. 3афиксируем $\varepsilon>0$. Для разбиения $\lambda \in \Pi_{N}$, обозначим

$$
f(\lambda)=\frac{(N-b(\lambda))(N-b(\lambda)-2) \cdots(N-b(\lambda)-2(n-1))}{N(N-1) \cdots(N-(n-1))} .
$$

Очевидно, что $f(\lambda) \rightarrow 1$ при $N \rightarrow \infty$ и $b(\lambda) / N \rightarrow 0$. Следовательно, существуют такие $N_{1} \in \mathbf{N}$ и $\delta>0$, что $1-\varepsilon \leqslant f(\lambda) \leqslant 1$ при $N \geqslant N_{1}$ и $b(\lambda) / N<\delta$. По лемме 5 существует такое $N \geqslant N_{1}$, что

$$
\bar{\mu}_{N}\left(A_{N}(\delta)\right) \geqslant 1-\varepsilon
$$


По лемме 4 , если $\lambda \in A_{N}(\delta)$, то $P(\lambda, u)=m_{n}(u)+R(\lambda, u)$, где $|R(\lambda, u)| \leqslant$ $\varepsilon$. Отсюда

$$
\mu_{n}(u)=m_{n}(u)+R_{1}+R_{2}-R_{3},
$$

где

$$
\begin{aligned}
& R_{1}=\sum_{\lambda \in A_{N}(\delta)} \bar{R}(\lambda, u) \bar{\mu}_{N}(\lambda) \leqslant \varepsilon \bar{\mu}_{N}\left(A_{N}(\delta)\right) \leqslant \varepsilon \\
& R_{2}=\sum_{\lambda \in \Pi_{N} \backslash A_{n}(\delta)} \bar{\mu}_{N}(\lambda) \bar{P}(\lambda, u) \leqslant \bar{\mu}_{N}\left(\Pi_{n} \backslash A_{N}(\delta)\right) \leqslant \varepsilon \\
& R_{3}=m_{n}(u)\left(1-\bar{\mu}_{N}\left(A_{N}(\delta)\right)\right) \leqslant \varepsilon .
\end{aligned}
$$

Следовательно, $\left|\mu_{n}(u)-m_{n}(u)\right| \leqslant 2 \varepsilon$, что завершает доказательство леммы 3.

6. Сведение к случаю насышенных мер. В этом пункте мы завершаем доказательство основной теоремы путем сведения ее к теореме 4.

Как было замечено в п. 3 , множества $\Sigma_{t}$ инвариантны относительно $\widehat{T}_{g}$ для всех $g \in \mathfrak{S}_{\infty}$. Случай $t=0$ тривиален, поскольку множество $\Sigma_{0}$ состоит из единственной точки. В дальнейшем мы предполагаем, что $t \neq 0$.

Из определения оператора $\widehat{T}_{g}$ и конструкции эргодических распределений $P^{x}$ следует, что при отождествлении $\Sigma_{t}$ со стандартным симплексом $\Sigma_{1}$ ограничение оператора $\widehat{T}_{g}, g \in \mathfrak{S}_{n}$, на $\Sigma_{t}$ принимает вид

$$
T_{g}^{t}=\Gamma_{t}^{-1}\left(\left.\widehat{T}_{g}\right|_{\Sigma_{t}}\right)=\sum_{k=0}^{n} t^{k}(1-t)^{n-k} \sum_{\substack{J \subset[n] \\|J|=k}} T_{\pi_{J}(g)} .
$$

Усредняя по $g \in \mathfrak{S}_{n}$, получаем

$$
\begin{aligned}
T_{n}^{t} & =\frac{1}{n !} \sum_{g \in \mathbb{S}_{n}} T_{g}^{t}=\sum_{k=0}^{n} t^{k}(1-t)^{n-k} \sum_{\substack{J \subset[n] \\
|J|=k}} \frac{1}{n !} \sum_{g \in \mathfrak{S}_{n}} T_{\pi_{J}(g)} \\
& =\sum_{k=0}^{n} t^{k}(1-t)^{n-k} \sum_{\substack{J \subset[n] \\
|J|=k}} \frac{1}{k !} \sum_{h \in \mathbb{S}[J]} T_{h} .
\end{aligned}
$$

Поскольку оператор $T_{g}$ зависит только от класса подстановки $g$, последняя нормированная сумма равна $T_{k}$. Следовательно,

$$
T_{n}^{t}=\sum_{k=0}^{n} t^{k}(1-t)^{n-k} \sum_{\substack{J C[n] \\
|J|=k}} T_{k}=\sum_{k=0}^{n}\left(\begin{array}{l}
n \\
k
\end{array}\right)(1-t)^{n-k} t^{k} T_{k} .
$$


Пусть $\nu$ есть $\left\{\widehat{T}_{g}\right\}$-инвариантная мера, и $\nu^{t}-$ условное распределение $\nu$ на $\Sigma_{t}$. Заметим, что $T_{0}^{t}=T_{1}^{t}=E$, где $E$ - тождественный оператор на $\Sigma_{1}$. В силу формулы $(10) T_{2}^{t}=t^{2} T_{2}+\left(1-t^{2}\right) E$. Поскольку мера $\bar{\nu}^{t}=\Gamma_{t}^{-1} \nu^{t}$ инвариантна относительно оператора $T_{2}^{t}$, то из последнего равенства следует, что она $T_{2}$-инвариантна. Рассуждая аналогичным образом, по индукции получаем, что мера $\bar{\nu}^{t}$ инвариантна относительно $\left\{T_{n}\right\}_{n=1}^{\infty}$. Из теоремы 4 следует, что $\bar{\nu}^{t}=P D(1)$ для любого $t \in[0,1]$, что завершает доказательство.

\section{СПИСОК ЛИТЕРАТУРЫ}

1. Вершик A.M. Описание инвариантных мер для действия некоторых бесконечных групп. - Докл. АН СССР, 1974, т. 218, № 4, с. 749-752.

2. Вершик A.M. Многозначные отображения с инвариантной мерой (полиморфизмы) и марковские операторы. - Зап. науч. сем. ЛОМИ, 1977, т. 72, с. 26-61.

3. Вершик A. М., Керов С. В. Локально полупростые алгебры. Комбинаторная теория и $K_{0}$ функтор. - Итоги науки и техники. Совр. проблемы матем., 1985 , т. 26, c. $3-56$.

4. Дынкин Е.Б. Классы эквивалентных случайных величин. - Успехи матем. наук, 1953, т. VII, с. 125-130.

5. Керов C. B. Комбинаторные примеры в теории $A F$-алгебр. - Зап. науч. сем. ЛОМИ, 1988 , т. 172 , с. $55-67$.

6. Керов С. В. Субординаторы и действия перестановок с квазиинвариантной мерой. - Зап. науч. сем. ПОМИ, 1995, т. 223, с. 181-218.

7. Керов С. В., Цилевич Н. В. Случайное дробление отрезка порождает виртуальные перестановки с распределением Ювенса. - Зап. науч. сем. ПОМИ, 1995, T. 223, c. $162-180$.

8. Цилевич Н.В. Распределение длин циклов бесконечных перестановок. - Зап. науч. сем. ПОМИ, 1995 , т. 223 , с. $148-161$.

9. Aldous D. Exchangeability and related topics. - Lecture Notes in Math., 1985, v. 1117 , p. $1-198$.

10. Furstenberg $H$. Boundary theory and stochastic processes on homogeneous spaces. - Harmonic Analysis on Homogeneous Spaces. Proc. of Symposia in Pure Mathematics. Ed. by C. C. Moore. Providence, RI: Amer. Math. Soc., 1973, p. 193232.

11. Kerov S. V., Olshanski G.I., Vershik A.M. Harmonic analysis on the infinite symmetric group. - C. R. Acad. Sci. Paris, 1993, v. 316, p. 773-778.

12. Kingman J.F.C. The representation of partition structures. - J. London Math. Soc., Ser. (2), 1978, v. 18, p. 374-380.

13. Kingman J.F.C. The coalescent. - Stochastic Process. Appl., 1982, v. 13, p. 235248.

14. Watterson G.A. The stationary distribution of the infinite-many neutral alleles diffusion model. - J. Appl. Probab., 1976, v. 17, p. 662-673.

Поступила в редакцию 15.IX.1998 